\title{
A Novel Role for Endocardium in Perinatal Valve Development: Lessons Learned from Tissue-Specific Gene Deletion of the Tie1 Receptor Tyrosine Kinase
}

\author{
Xianghu Qu and H. Scott Baldwin
}

\begin{abstract}
The mechanisms regulating late-gestational and early postnatal semilunar valve remodeling and maturation are poorly understood. Tie1 is a receptor tyrosine kinase with broad expression in embryonic endothelium. During semilunar valve development, Tie1 expression becomes restricted to the turbulent, arterial surfaces of the valves in the perinatal period. Previous studies in our laboratory have demonstrated that Tie1 can regulate cellular responses to blood flow and shear stress. We hypothesized that Tie1 signaling would regulate the flowdependent remodeling of the semilunar valves associated with the conversion from maternal/placental to independent neonatal circulation. To circumvent the embryonic lethality of the Tie 1 null mutation, we developed a floxed Tie1 allele and crossed it with an $\mathrm{Nfactcl}^{\text {en }} \mathrm{Cre}$ line that mediates gene excision exclusively in the endocardial cushion endothelium. Excision of Tie 1 resulted in aortic valve leaflets displaying hypertrophy with perturbed matrix deposition. The valves demonstrated insufficiency and stenosis by ultrasound, and atomic force microscopy documented decreased stiffness in the mutant aortic valve consistent with an increased glycosaminoglycan to collagen ratio. These data suggest that active endocardial to mesenchymal signaling, at least partially mediated by Tie1, is uniquely required for normal remodeling of the aortic but not pulmonary valve in the late gestation and postnatal animal.
\end{abstract}

\footnotetext{
X. Qu $(\bowtie)$

Department of Pediatrics (Cardiology), Vanderbilt University, 2213 Garland Ave, Nashville, TN 37232-0493, USA

e-mail: xianghu.qu@vanderbilt.edu
}

H.S. Baldwin

Department of Pediatrics (Cardiology), Vanderbilt University, 2213 Garland Ave, Nashville, TN 37232-0493, USA

Department of Cell and Developmental Biology, Vanderbilt University, 2213 Garland Ave, Nashville, TN 37232-0493, USA

T. Nakanishi et al. (eds.), Etiology and Morphogenesis of Congenital Heart Disease, DOI 10.1007/978-4-431-54628-3_19 


\section{Keywords}

Semilunar valves • Endocardium • Extracellular matrix • Tie1

\subsection{Introduction}

Cardiovascular defects are the most common congenital abnormality in the human population, affecting approximately 1 out of every 100 live births worldwide [1]. Defects in valve development account for 25-30\% of these malformations. Therefore, there is significant interest in understanding the mechanisms that underlie the complex process of heart valve development. During heart valve formation, a subset of endothelial cells overlying the future valve site are specified to delaminate, differentiate, and migrate into the cardiac jelly, a process referred to as endothelial-mesenchymal transformation or transdifferentiation (EMT). Locally expanded swellings of cardiac jelly and mesenchymal cells are referred to as cardiac cushions. In a poorly understood process, these cardiac cushions undergo extensive remodeling from bulbous swellings to eventual thinly tapered heart valve leaflets [2]. Numerous studies have focused on identifying the major regulators of valve development using murine and avian embryos and have particularly focused on the early stages of valve development; however, the pathways regulating the final events of remodeling and maturation have not been well defined.

The orphan receptor tyrosine kinase Tie1 is primarily expressed in endothelial cells and is closely related to Tie2, the receptor for the angiopoietins. Both Tie 1 and Tie2 are essential for developmental vascularization where they appear to have roles in promoting microvessel maturation and stability. Targeted disruption of Tie1 gene in mice results in a lethal phenotype between E14.5 and P0 characterized by extensive edema, hemorrhage, and defective microvessel integrity [3-5]. Expression of Tie1 is first detected in the endothelium of mice at E8.0, and by E12.5, Tie1 is robustly expressed in the valvular endothelium, vasculature, heart, and lungs [69]. Tie1 expression subsides in the postnatal period, although low levels of Tie1 persist into adulthood on the arterial side of the valve leaflet as well as branch points of the descending aorta [10]. Thus, Tie1 is expressed in the vasculature, valves, and other endothelial cell populations in the developing heart during critical periods of valve morphogenesis. However, its potential roles in heart valve development are totally unknown. Here, using a conditional knockout mouse model, we show that lack of Tie1 in endocardial cells leads to hypertrophic semilunar valves in the postnatal and adult heart.

\subsection{Model for Valvar Endocardial-Specific Gene Deletion}

To specifically determine the potential role of Tie1 in valve morphogenesis, we have developed a novel valve endocardial cell-specific Cre mouse line ( $\mathrm{Natcl} \mathrm{C}^{\text {en }} \mathrm{Cre}$ ). We previously identified a transcriptional enhancer that regulates the sustained expression of $\mathrm{Nfatcl}$ exclusively in the endocardium overlying the 
endocardial cushions [11]. We then utilized this enhancer to develop a transgenic mouse line, $\mathrm{Nfactcl} \mathrm{I}^{\text {en }} \mathrm{Cre}$, which has recently been described by our lab [12]. To determine the utility and efficiency of this model for deletion at latter stages of cardiac development, we have analyzed Cre expression in the developing valves by lacZ staining and qRT-PCR of $\mathrm{Nfactcl}^{\text {en }}$ Cre transgenic mice bred with R26R reporter mice (Fig. 19.1a-d). lacZ staining confirmed that Cre activation is first detected at E9.5 in the developing AVC region and in OFT region by E10.5 (data not shown). Cre activation is localized to endocardial cushion endocardium of the outflow track at E12.5 and at E14.5 (Fig. 19.1a, b) and remains restricted to the endocardium of overlying the developing valves throughout gestation into the perinatal period (Fig. 19.1c) and in the adult (data not shown). To determine the utility of $\mathrm{Nactcl} \mathrm{I}^{\text {en }} \mathrm{Cre}$ for tissue-specific deletion, we crossed these mice with our mice harboring a floxed Tie1 allele previously described [5]. When Tie1 expression as determined by QT-PCR was normalized to expression detected in the adult animal, we observed the normal attenuation of Tie1 previously described by our group [5]. In addition we observed significant attenuation of Tie1 expression as a result of Cre-mediated deletion in the heart that was not detected in other vascular beds (data not shown). Thus our Cre transgenic line $\mathrm{Nfactcl}^{\text {en }} \mathrm{Cre}$ mediates loxP excision exclusively in the endocardium overlying the endocardial cushions and not in mesenchyme derived from EMT or other endothelial populations.

\subsection{Tie1 Is Required for Late-Gestational and Early Postnatal Aortic Valve Remodeling}

To investigate the potential role of Tie1 in valve morphogenesis, we generated endocardial-specific Tiel knockout (Tiel ${ }^{f / l z}$; Nfactcl ${ }^{\text {en }} \mathrm{Cre}$ ) mice by breeding mice homozygous for a floxed Tie1 allele [5] with mice heterozygous for a null mutation in Tie $1^{\text {fllz }}$ (Tie $1^{+/ l z}$ is a "knock-in"/knockout" insertion of lacZ into the Tie 1 locus [3]) as well as the $N$ factcl ${ }^{\text {en }}$ Cre transgene. Analysis of timed matings revealed that no embryos died in utero during early gestation. However, within a few days after birth, Tiel ${ }^{f / l z}$; Nfactcl ${ }^{\text {en }}$ Cre pups could be distinguished from Tiel ${ }^{\text {fl/fl }}$ or Tiel $1^{\text {fllz }}$ littermates as the mutants were often growth retarded (Fig. 19.2) and lethargic, both of which are characteristics of human patients with heart defects. Most of mutants did not survive to adulthood. Histological analysis revealed no significant differences in valve formation between mutants and littermates until E16.5 (Fig. 19.3a, b), indicating early events of valvulogenesis occur normally in Tiel mutant mice. However, an increase in semilunar valve size was noted in Tie $^{f / l z}$; $\mathrm{Nfactcl}^{\text {en }}$ Cre mice from E18.5 (Fig. 19.3c, d) and early perinatal periods with the discrepancy in valve size continuing into the adult (Fig. 19.3e, f). Interestingly, the valve abnormalities were not only limited to the late gestation and postnatal period, but they were also primarily only detected in the aortic valves. The aortic valve areas of adult Tie ${ }^{f / l z}$;Nfactcl ${ }^{\text {en }} \mathrm{Cre}$ mice were nearly twice as large as Tie ${ }^{f l / f}$ aortic valves (increased by $88.3 \%$ ). The pulmonary valves of Tie ${ }^{f / l l z}$; Nfactcl ${ }^{\text {en }}$ Cre mice were somewhat thicker than the controls, but the difference was not significant. There were no differences in cell proliferation or apoptosis between mutant mice 
A

Nfatc1 ${ }^{\text {en }}$ Cre

HSP68 nlsCre-pA Nfatc1-enhancer
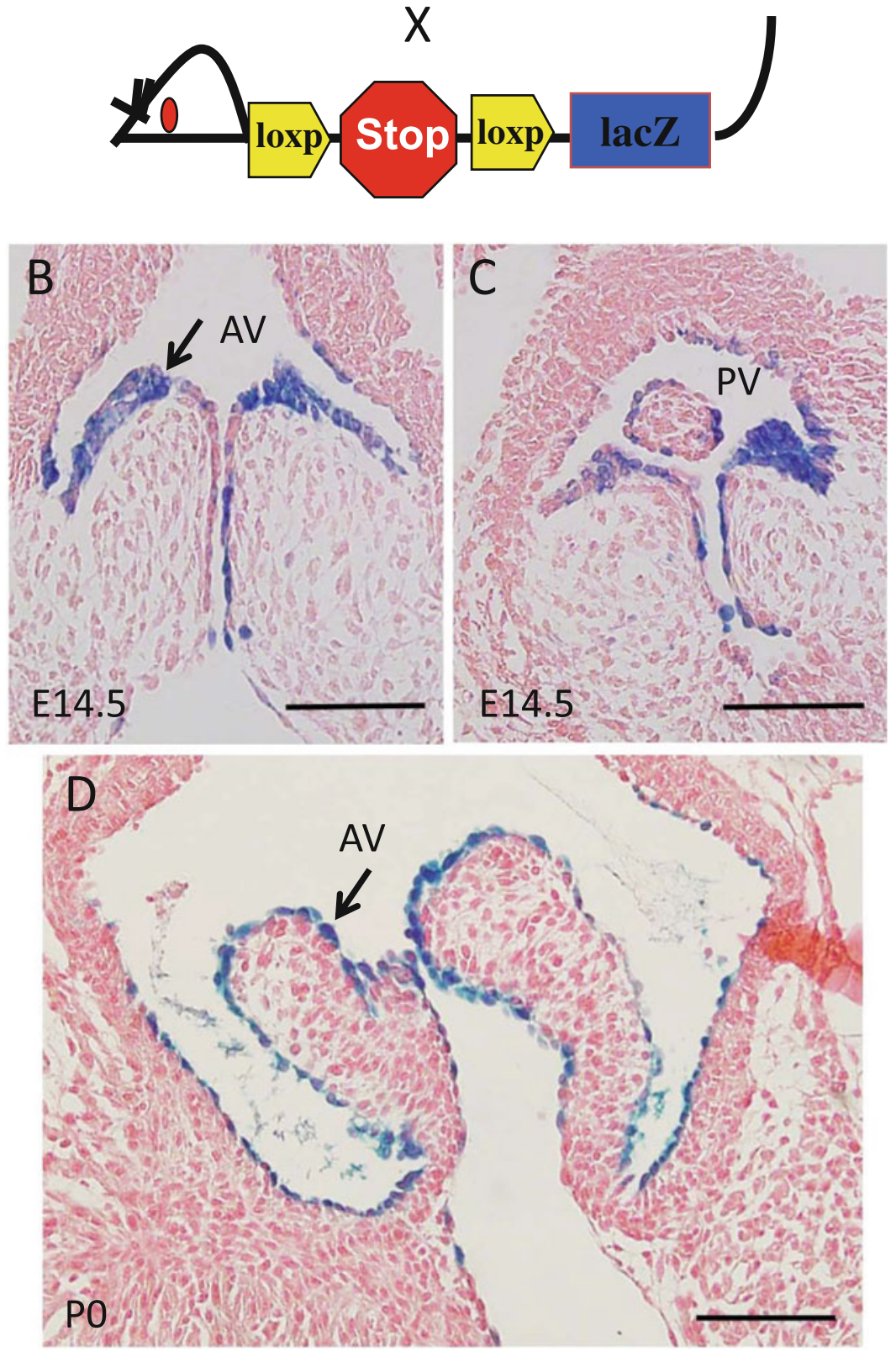

Fig. 19.1 $\mathrm{Nfactcl}^{\text {en }} \mathrm{Cre}$ mediates loxP excision exclusively in the valvular endocardium. (a) Schematic of breeding of NfatclenCre and R26fslz mice. X-gal staining of R26 fslz $\mathrm{Nfactcl}^{\text {en }} \mathrm{Cre}$ heart sections shows that expression of the Cre is restricted to the endothelium of the developing 
Fig. 19.2 Tie ${ }^{f l / z}$; Nfactcl ${ }^{\text {en }} \mathrm{Cre}$ animals are smaller than the controls

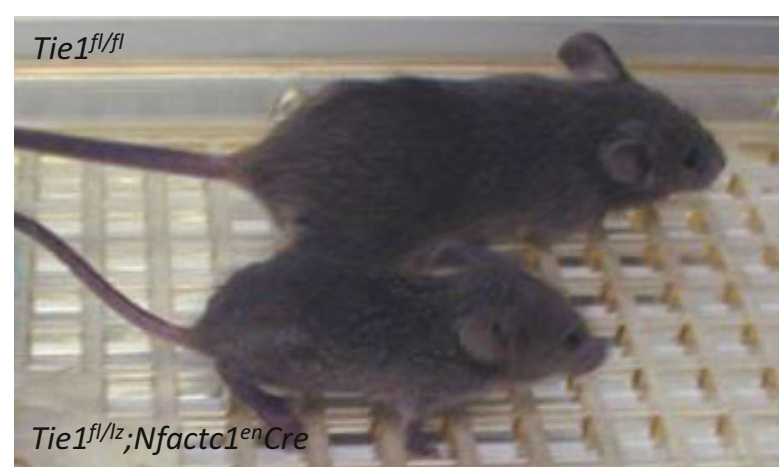

and littermates suggesting a difference in extracellular matrix (ECM) composition or production. Consistent with this, Movat's pentachrome stain revealed an increase in proteoglycan/glycosaminoglycan (GAGs) production and/or degradation in the valve interstitium of postnatal Tiel ${ }^{f / l z}$; Nfactcl ${ }^{\text {en }} \mathrm{Cre}$ mice. As compared to Tie $\mathrm{I}^{\text {flfl }}$, GAGs content (Fig. 19.3e, f) was increased and total collagen content (data not shown) was decreased in aortic valve leaflets of Tie ${ }^{\text {flllz }}$;Nfactcl ${ }^{\text {en }}$ Cre animals. Thus, attenuation of Tie1 results in severe abnormalities in ECM remodeling that are characteristic of critical events in late gestation and postnatal valve development in chicken, mouse, and humans $[13,14]$. This work suggests that there is a non-cell autonomous defect that results from endocardial-specific Tie1 deletion as the ECM is produced primarily from the valvular interstitial cells (VICS), not the endocardium. This work also provides in vivo support for the in vitro observations that valvular endocardial cells regulate the phenotype of VICS. In addition, Tie1 mutant aortic valves demonstrated a decrease in rigidity as measured by atomic force microscopy and valvular insufficiency, as determined by high-resolution ultrasound (data not shown).

\subsection{Future Directions}

This study describes a unique dosage-dependant role for Tie1 during later stages of valve remodeling. Tie 1 is essential for valve remodeling, and abnormalities within late-gestational valve remodeling lead to flaccid valve leaflets, which in turn do not function correctly in the animal. It is likely that the mortality observed in Tie $1^{f / l z}$; Nfactcl ${ }^{\text {en }}$ Cre mice can be attributed to aortic insufficiency and ultimate heart failure. However, the mechanisms regulating late-gestational and early postnatal semilunar valve remodeling and maturation are still poorly understood.

Fig. 19.1 (continued) valves (arrowhead) at E14.5 (b, c) and at P0 (d), and weak lacZ expression remains in the majority of the valvular endothelial cells through adulthood (d). HSP68 indicates heat shock protein $68 ; A V$ aortic valve, $P V$ pulmonary and aortic valve. Scale bars: $50 \mu \mathrm{m}$ 


\section{Tie $1^{f l / f l}$}
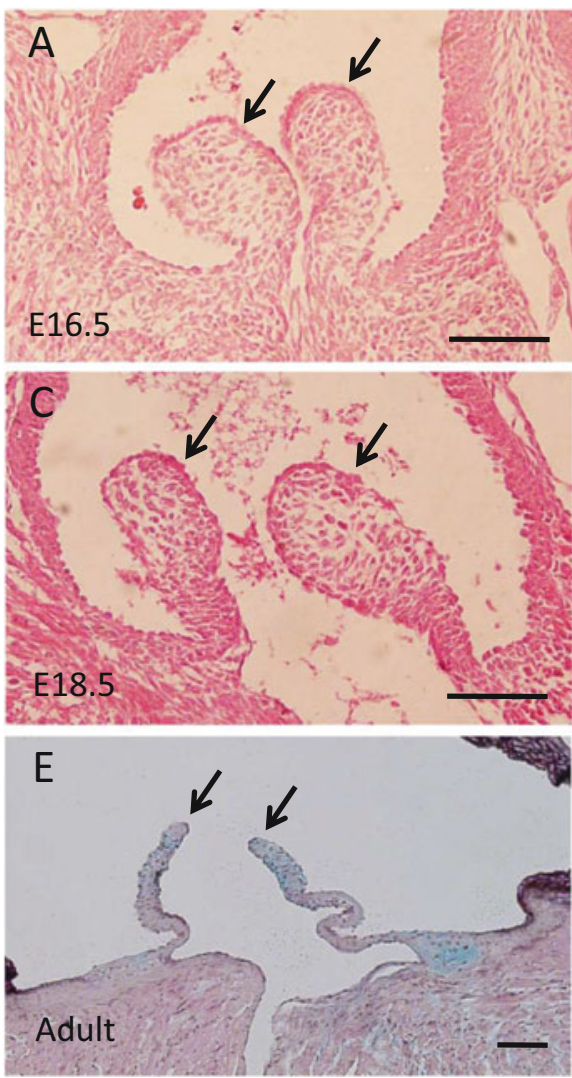

Fig. 19.3 Tie1 attenuation leads to enlarged aortic valves and abnormal ECM deposition and organization. H\&E-stained sections of valves of Tie $1^{f l / f t}$ and Tie ${ }^{f l / l z} ;$ Nfactcl $^{\text {en }} \mathrm{Cre}$ mice at E16.5 (a, b) and at E18.5 (c, d). Compared to Tie ${ }^{f l f t}$ mice, Tie $1^{f l / z} ; N$ factcl $I^{\text {en }}$ Cre mice have enlarged aortic valves. Movat's pentachrome stain shows enlarged aortic valves with increased GAGs deposition (blue) in adult ( 9 weeks) Tie ${ }^{f l / l z} ; \mathrm{Nfactcl}^{\text {en }} \mathrm{Cre}$ mice (f) as compared to the controls (e). Arrows indicate valve leaflets. Scale bars: $50 \mu \mathrm{m}$

Tie1, although a close sequence homologue of Tie2, does not interact directly with the angiopoietins, and its in vivo ligands are yet to be identified. Nevertheless, growing reports based on in vitro studies suggest that a primary function of Tie1 might be to modulate Tie2 signaling and function [15-17]. Furthermore, both Tie1 and Tie 2 are co-expressed in endocardial cells from very early endocardial cushion stage to the mature valve stage. So, we hypothesize that Tie1 signals independent of Tie 2 and also acts an inhibitory co-receptor for Tie2 activation. Further investigation on this project using double (Tie1 and Tie2) conditional knockouts is currently on the way. Additional animal models are being developed to characterize the mechanism of Tie1-Tie2 interactions in modulating critical events in cardiac ontogeny in vivo, and expression profiling is being implemented to delineate 
downstream signaling cascades that are activated by Tie1 and Tie1-Tie2 interactions. These studies will be essential for understanding the role of the endocardium in modulation valve matrix deposition and remodeling in an effort to unravel basic mechanisms of congenital heart disease.

Acknowledgements This work was supported by grant from NHLB/NIH: RL1HL0952551 (H.S.B.)

Open Access This chapter is distributed under the terms of the Creative Commons AttributionNoncommercial 2.5 License (http://creativecommons.org/licenses/by-nc/2.5/) which permits any noncommercial use, distribution, and reproduction in any medium, provided the original author(s) and source are credited.

The images or other third party material in this chapter are included in the work's Creative Commons license, unless indicated otherwise in the credit line; if such material is not included in the work's Creative Commons license and the respective action is not permitted by statutory regulation, users will need to obtain permission from the license holder to duplicate, adapt or reproduce the material.

\section{References}

1. Hoffman JI, Kaplan S. The incidence of congenital heart disease. J Am Coll Cardiol. 2002;39:1890-900.

2. DeLaughter DM, Saint-Jean L, Baldwin HS, et al. What chick and mouse models have taught us about the role of the endocardium in congenital heart disease. Birth Defects Res Part A Clin Mol Teratol. 2011;91:511-25.

3. Puri MC, Rossant J, Alitalo K, Bernstein A, Partanen J. The receptor tyrosine kinase tie is required for integrity and survival of vascular endothelial cells. EMBO J. 1995;14:5884-91.

4. Sato TN, Tozawa Y, Deutsch U, Wolburg-Buchholz K, Fujiwara Y, Gendron-Maguire M, Gridley T, Wolburg H, Risau W, Qin Y. Distinct roles of the receptor tyrosine kinases tie-1 and tie-2 in blood vessel formation. Nature. 1995;376:70-4.

5. Qu X, Tompkins K, Batts LE, Puri M, Baldwin S. Abnormal embryonic lymphatic vessel development in tie1 hypomorphic mice. Development. 2010;137:1285-95.

6. Partanen J, Armstrong E, Makela TP, Korhonen J, Sandberg M, Renkonen R, Knuutila S, Huebner K, Alitalo K. A novel endothelial cell surface receptor tyrosine kinase with extracellular epidermal growth factor homology domains. Mol Cell Biol. 1992;12:1698-707.

7. Sato TN, Qin Y, Kozak CA, Audus KL. Tie-1 and tie-2 define another class of putative receptor tyrosine kinase genes expressed in early embryonic vascular system. Proc Natl Acad Sci U S A. 1993;90:9355-8.

8. Dumont DJ, Fong GH, Puri MC, Gradwohl G, Alitalo K, Breitman ML. Vascularization of the mouse embryo: a study of flk-1, tek, tie, and vascular endothelial growth factor expression during development. Dev Dyn. 1995;203:80-92.

9. Taichman DB, Schachtner SK, Li Y, Puri MC, Bernstein A, Scott Baldwin H. A unique pattern of tie1 expression in the developing murine lung. Exp Lung Res. 2003;29:113-22.

10. Woo KV, Qu X, Babaev VR, Linton MF, Guzman RJ, Fazio S, Baldwin HS. Tie1 attenuation reduces murine atherosclerosis in a dose-dependent and shear stress-specific manner. J Clin Invest. 2011;121:1624-35.

11. Zhou B, Wu B, Tompkins KL, Boyer KL, Grindley JC, Baldwin HS. Characterization of nfatc1 regulation identifies an enhancer required for gene expression that is specific to pro-valve endocardial cells in the developing heart. Development. 2005;132:1137-46. 
12. Wu B, Wang Y, Lui W, Langworthy M, Tompkins KL, Hatzopoulos AK, Baldwin HS, Zhou B. Nfatc1 coordinates valve endocardial cell lineage development required for heart valve formation. Circ Res. 2011;109:183-92.

13. Hinton Jr RB, Lincoln J, Deutsch GH, Osinska H, Manning PB, Benson DW, Yutzey KE. Extracellular matrix remodeling and organization in developing and diseased aortic valves. Circ Res. 2006;98:1431-8.

14. Aikawa E, Whittaker P, Farber M, Mendelson K, Padera RF, Aikawa M, Schoen FJ. Human semilunar cardiac valve remodeling by activated cells from fetus to adult: implications for postnatal adaptation, pathology, and tissue engineering. Circulation. 2006;113:1344-52.

15. Saharinen P, Kerkela K, Ekman N, Marron M, Brindle N, Lee GM, Augustin H, Koh GY, Alitalo K. Multiple angiopoietin recombinant proteins activate the tie1 receptor tyrosine kinase and promote its interaction with tie2. J Cell Biol. 2005;169:239-43.

16. Marron MB, Hughes DP, Edge MD, Forder CL, Brindle NP. Evidence for heterotypic interaction between the receptor tyrosine kinases tie-1 and tie-2. $\mathrm{J}$ Biol Chem. 2000;275:39741-6.

17. Seegar TC, Eller B, Tzvetkova-Robev D, Kolev MV, Henderson SC, Nikolov DB, Barton WA. Tie1-tie2 interactions mediate functional differences between angiopoietin ligands. Mol Cell. 2010;37:643-55. 